\title{
Optimizing intrinsic and extrinsic process factors to minimize folate loss in fruit and vegetables
}

\author{
OEY, I; VERLINDE, P.; NGUYEN, M.T.; ARROQUI, C.*; HENDRICKX, M.; VAN \\ LOEY, A.
}

Faculty of Bioscience Engineering, Centre for Food and Microbial Technology, Laboratory of Food Technology, Katholieke Universiteit Leuven, Kasteelpark Arenberg 22, B-3001 Leuven, Belgium.

*Area de Tecnologia de Alimentos, Universidad Publica de Navarra, Campus Arrosadía s/n, 31005, Pamplona, Spain

Mailing address for corresponding author: Indrawati.Hartono@biw.kuleuven.be

\section{INTRODUCTION}

Folates (vitamin B family) play an important role in the metabolism of amino and nucleic acids. An inadequate folate intake can cause several disorders, cancers and diseases. Evidences have shown that food processing either thermal or non thermal such as high-pressure/HP applied to (plant based) foods have an influence on the stability of (natural) folates. Hereto, this research aimed at the optimization of the intrinsic $(\mathrm{pH}$, antioxidant, oxygen) and extrinsic (temperature/T, pressure/P, time/t) process factors to minimize the loss of endogenous folate in fruit and vegetables during processing.

\section{MATERIALS AND METHODS}

Study on folate stability in model system

Temperature and pressure stability of natural monoglutamate-folates (5-methyltetrahydrofolate (5MTHF), 5-formyl-tetrahydrofolate (5FTHF) and tetrahydrofolate (THF)) in different model systems (i.e. differences in $\mathrm{pH}$ 's, buffer compositions, oxygen concentrations, ascorbic acid/L-AA ratio's) was studied on a kinetic basis. The samples were subjected to isothermal-isobaric treatments for predefined time intervals in thermostated small sample holders or in multivessel-HP equipment. The folate and ascorbic acid concentrations were measured using RP-HPLC method.

\section{Study on folate stability in intact food matrices}

For validation, folate stability in intact vegetables i.e. carrots, asparagus and Brussels sprouts was studied. Throughout the study, a single batch of vegetables was used. The samples were packed in stainless steel tubes and in polyethylene plastic bags respectively for thermal and pressure treatments.

Prior to folate identification and quantification, folate extraction using tri enzyme (amylase, conjugase and protease) and heating extraction was conducted. Afterwards, folate purification using folate binding protein affinity chromatography was carried out. The folate concentrations was measured using RP-HPLC methods. 


\section{RESULTS AND DISCUSSION}

In this research, effects of intrinsic and extrinsic factors on folate stability during food processing were investigated. Study on folate stability in model systems showed that (i) the extent of folate degradation depended on oxygen concentration/content $\left(\left[\mathrm{O}_{2}\right]\right)$; (ii) L-AA increased folate stability; (iii) the molar ratio between L-AA or folate and the initial $\left[\mathrm{O}_{2}\right]$ was an important parameter to determine the proportion of aerobic degradation; (iv) the minimum L-AA concentration needed to prevent folate oxidation was at least $2 \mathrm{x}$ the initial $\left[\mathrm{O}_{2}\right]($ Oey et al., 2006) and (v) the order of folate stability was 5 FTHF $>5$ MTHF $>$ THF (Nguyen et al., 2005).

Based on kinetic data, the oxidation rate was accelerated by (i) increasing pressure and temperature and (ii) lowering the $\mathrm{pH}$. Temperature of folate oxidation at $0.1 \mathrm{MPa}$ was shifted to lower temperatures when applying pressure ( $>50 \mathrm{MPa})$ (because of negative activation volume). As compared to conventional thermal processing, the folate stability could also be affected during HP because oxidation reaction was enhanced (Nguyen et al. 2003, 2004, 2006; Indrawati et al. 2004, 2005, . In addition, validation studies in food matrices i.e. in fruit and vegetables showed that folate degradation in intact fruit and vegetables (taking into account folate loss due to leaching for thermal treatment) was low because of the existence of endogenous L-AA (Indrawati et al., 2004).

\section{CONCLUSIONS}

Folate loss can be limited by adding antioxidant or minimizing dissolved $\left[\mathrm{O}_{2}\right]$. Regarding to folate loss in fruit and vegetables due to leaching, HP can be an interesting alternative because most of HP-treated food products are vacuum-packed and no contact with pressure medium. Furthermore, HP has no/little effects on covalent bonds resulting in limited loss e.g. in vitamin content, color, taste etc.

\section{REFERENCES}

Indrawati, Arroqui, C., Messagie, I., Nguyen, M.T., Van Loey, A., Hendrickx, M. 2004. Comparative study on pressure and temperature stability of 5methyltetrahydrofolic acid in model systems and in food products. Journal of Agricultural and Food Chemistry, 52, 485-492.

Indrawati, Van Loey, A., Hendrickx. 2005. Pressure and temperature stability of 5methyltetrahydrofolic acid: A kinetic study. Journal of Agricultural and Food Chemistry 53(8): 3081-3087.

Nguyen, M.T., Indrawati, Hendrickx, M.E. 2003. Model studies on the stability of folic acid and 5-methyltetrahydrofolic acids degradation during thermal treatment in combination with high hydrostatic pressure. Journal of Agricultural and Food Chemistry, 51(11), 3352-3357. 
Nguyen, M.T., Indrawati, Van Loey, A., Hendrickx, M. 2004. Effect of pH on temperature stability of folates. Comm. Appl. Biol. Sci. Ghent University, 69/2. pp 203-206.

Nguyen, M.T., Oey, I., Hendrickx, M., Van Loey, A. 2006. Kinetics for isobaricisothermal degradation of $(6 \mathrm{R}, \mathrm{S})$ 5-formyltetrahydrofolic acid in a model system. European Food Research and Technology, 219(2), 161-166.

Oey, I., Verlinde, P., Hendrickx, M. Van Loey, A. 2006. Temperature and pressure stability of L-ascorbic acid and/or [6s] 5-methyltetrahydrofolic acid: a kinetic study. European Food Research and Technology, 223: 71-77. 